\title{
Article \\ Numerical Modeling of Nondestructive Testing of Various Conductive Objects inside Metal Enclosures Using ELF/VLF Magnetic Fields
}

\author{
Hoyoung Kim * ${ }^{-1}$ and Vijay Harid \\ Department of Electrical Engineering, University of Colorado Denver, Denver, CO 80204, USA; \\ vijay.harid@ucdenver.edu \\ * Correspondence: hoyoung.kim@ucdenver.edu
}

Citation: Kim, H.; Harid, V.

Numerical Modeling of

Nondestructive Testing of Various

Conductive Objects inside Metal

Enclosures Using ELF/VLF Magnetic

Fields. Appl. Sci. 2021, 11, 3665.

https://doi.org/10.3390/app11083665

Academic Editor:

Giuseppe Lacidogna

Received: 9 March 2021

Accepted: 17 April 2021

Published: 19 April 2021

Publisher's Note: MDPI stays neutral with regard to jurisdictional claims in published maps and institutional affiliations.

Copyright: (c) 2021 by the authors. Licensee MDPI, Basel, Switzerland. This article is an open access article distributed under the terms and conditions of the Creative Commons Attribution (CC BY) license (https:// creativecommons.org/licenses/by/ $4.0 /)$
Featured Application: ELF/VLF magnetic fields is a promising tool for nondestructive testing of various conductive objects within shielded structures.

Abstract: Nondestructive evaluation of various conductive objects through metal enclosures is investigated by using ELF/VLF magnetic induction fields in detailed simulations. ELF/VLF magnetic fields $(<30 \mathrm{kHz}$ ) have a unique ability to penetrate highly conductive or permeable shields. Using a magnetic dipole source antenna, objects hidden inside a metal enclosure are imaged via examining distortions to the field outside the enclosure. The field distortion is parametrically studied by varying the size, conductivity, and permeability of the hidden objects. Furthermore, the importance of the conductivity of the enclosure itself is investigated using both low $\left(10^{6} \mathrm{~S} / \mathrm{m}\right)$ and high $\left(10^{8} \mathrm{~S} / \mathrm{m}\right)$ conductivity metallic shields. It is shown that the responses are quite sensitive to the object and shield parameters; both qualitative and quantitative properties of the field distortions are described in detail. The simulation results suggest that properties of hidden conductive or permeable objects, over a relatively wide range of parameters (both geometry and material), can be inferred nondestructively using ELF/VLF magnetic induction fields.

Keywords: extremely and very low frequency; electromagnetic near field; nondestructive evaluation; induced surface current

\section{Introduction}

Extremely and very low frequency (ELF/VLF) signals $(<30 \mathrm{kHz})$ have been widely employed over several decades for radio waves, submarine communications, geophysical prospecting, and upper atmospheric remote sensing [1-6]. The primary reason is that ELF/VLF signals can propagate long distances and penetrate through conductive barriers [7]. In previous works, ELF/VLF magnetic fields have been demonstrated to be a viable source for imaging conductive objects that are obscured by metal shields $[7,8]$. However, the relative sensitivity of through-shield imaging on both material and geometric properties of hidden objects remains an unexplored area of investigation. As such, we parametrically investigate the sensitivity of nondestructive ELF/VLF testing using simulations of objects with different material properties and geometries.

A typical methodology for testing conductive objects using magnetic fields utilizes an array of several inductive coils that surrounds the objects [9-11]. The inductive coils generate magnetic fields and are applied at frequencies in the range of $1-30 \mathrm{MHz}[9,11,12]$. One of the coils is excited, while the other coils are treated as receivers. The applied electromagnetic fields induce Eddy currents on conductive objects. Eddy currents lead to scattering magnetic fields with a depletion region [13]. Based on the receiver measurements, image reconstruction can be performed with various inversion algorithms such as LASSO, Ridge regression, elastic-net, and the Gauss-Newton method [14-16]. Although magnetic 
induction-based inversion has been employed by several researchers, few previous works have considered nondestructive testing of metallic objects that are surrounded by a highly conductive shield $\left(10^{6} \mathrm{~S} / \mathrm{m}<\sigma<10^{8} \mathrm{~S} / \mathrm{m}\right)$ [8]. In real materials, nickel $\left(1.28 \times 10^{6} \mathrm{~S} / \mathrm{m}\right)$, aluminum $\left(3.5 \times 10^{7} \mathrm{~S} / \mathrm{m}\right)$, copper $\left(5.8 \times 10^{7} \mathrm{~S} / \mathrm{m}\right)$, and silver $\left(6.3 \times 10^{7} \mathrm{~S} / \mathrm{m}\right)$ are in the range of high conductivity [17]. The purpose of this work is to extend the body of literature and consider the viability of ELF/VLF magnetic fields for the nondestructive evaluation of shielded objects with realistic material and geometric properties. Section 2 discusses analytical approximations for understanding basic properties of ELF/VLF field distortions due to conductive objects inside shielded enclosures. Section 3 utilizes numerical simulations to investigate the impact of material properties and geometry. Section 4 provides a conclusion and discussion of the results with implications for future work.

\section{Theoretical Background}

Evaluating ELF/VLF magnetic fields as a nondestructive modality will in general require a numerical approach. However, analytical approximations can be utilized to provide a high-level understanding of the important features that are present in the problem. Thus, this section provides closed form expressions that serve as a guide for interpreting results from more sophisticated simulations in Section 3. A magnetic shielding effect is dependent on the complex amplitude of the induced dipole moment. The real part of the induced dipole moment is investigated as a function of relative permeability, the radius of the object, and source frequency. All electromagnetic quantities including the induced dipole moments and magnetic fields are assumed to be time harmonic $\left(e^{j w t}\right)$. All data are calculated when the source field has zero phase $(t=0)$.

Figure 1 shows a simplified geometry for a conductive object and a metal enclosure immersed in a z-directed uniform magnetic field $(H)$. The object is a sphere with radius $R$ and the thin enclosure is a metal sphere with radius $R_{\mathrm{s}}$ radius and thickness $d$. It is assumed that the thickness is small compared to a skin depth of an incident field. The quantity $H_{0}$ represents the amplitude of the illuminating magnetic field. In Figure 1a, the object is illuminated by the $H$ field, and the field induces a conduction current and magnetic polarization [7]. The physical process can be equivalently modeled as an induced magnetic dipole $\left(m_{p}\right)$ given by Equation (1).

$$
m_{p}=-2 \pi R^{3} A_{1} H_{0}
$$



(a)

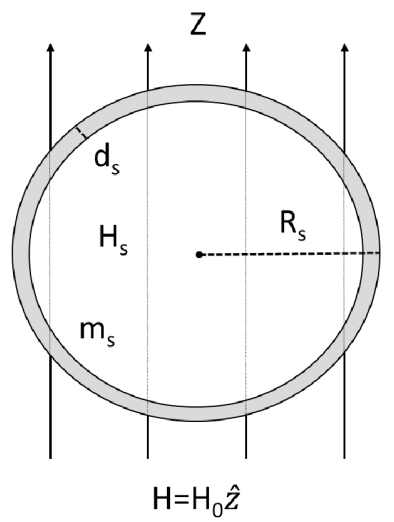

(b)

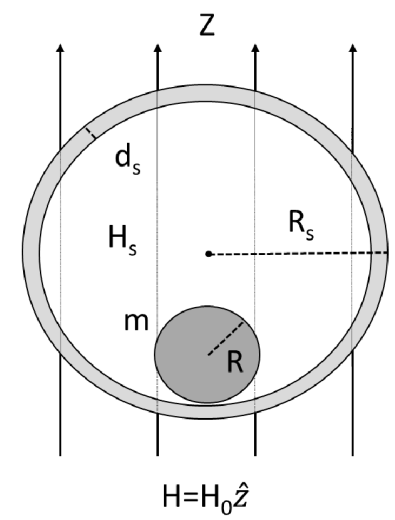

(c)

Figure 1. The geometry of spherical (a) conductive object, (b) thin enclosure, and (c) hidden spherical object in thin enclosure using z-directed uniform magnetic field with $H_{0}$ amplitude. An inside conductive object has $R$ radius and induces magnetic dipole $\left(m_{p}\right)$. The spherical shell has an $R_{\mathrm{s}}$ outer radius and $d$ thickness. The incident field $(H)$ induces magnetic dipole $\left(m_{s}\right)$ and is scattered $\left(H_{S}\right)$. 
The quantity $A_{1}$ is given by Equation (2) where $k$ and $\mu$ are complex wave number and permeability [17]. The quantity $k$ is calculated by Equation (3) where $\epsilon_{0}, \epsilon_{r}, \mu_{0}, \mu_{r}, \sigma$, and $\omega$ are the permittivity of free space, relative permittivity, permeability of free space, relative permeability, conductivity, and frequency. The quantities $\epsilon_{0}$ and $\mu_{0}$ are $8.854 \times 10^{-12} \mathrm{~F} / \mathrm{m}$ and $1.257 \times 10^{-6} \mathrm{H} / \mathrm{m}$ respectively for free space.

$$
\begin{gathered}
A_{1}=X+j Y=\frac{\left(1+(k R)^{2}+2 \mu_{r}\right) \sinh (k R)-\left(2 \mu_{r}+1\right) k R * \cosh (k R)}{\left(1+(k R)^{2}-\mu_{r}\right) \sinh (k R)+\left(\mu_{r}-1\right) k R * \cosh (k R)}, \\
k=\omega \sqrt{\mu_{0} \mu_{r}\left(\epsilon_{0} \epsilon_{r}-j \frac{\sigma}{\omega}\right)}
\end{gathered}
$$

In Equation (2), $X$ and $Y$ represent an in-phase (real) and quadrature (imaginary) components, respectively [17]. The $Y$ quantity can be interpreted as the out-of-phase component of the dipole moment and determines how strongly the induced dipole moment's phase will differ from that of the incident field. Equation (1) is valid when the sphere is small enough and the injected magnetic field is uniform across the sphere. Material properties such as conductivity and permeability induce an in-phase response with an amplitude and a polarity. For example, the in-phase response of conductive metals (e.g., copper and aluminum; $\mu_{r}=1$ ) is always positive, while ferrous metals (e.g., purified iron, permalloy; $\mu_{r}>1$ ) exhibit negative in-phase behavior as a function of frequency [17].

In contrast to the solid object, the thin-shell shield (as shown in Figure 1b) can be modeled via an induced magnetic dipole moment $\left(m_{s}\right)$ that is added to the field response of the isolated object. The moment is calculated by Equations (4) and (5). The quantities $d_{s}$ and $R_{s}$ are the thickness and radius of the shell respectively [18]. The quantities $A_{2}$ and $\alpha$ are calculated by Equations (5) and (6) where $\mu_{s}$ and $\sigma_{s}$ are the permeability and conductivity of the shell.

$$
\begin{gathered}
m_{s}=-2 \pi R_{s}^{3} A_{2} H_{0} \\
A_{2}=-\frac{2 \mu_{s}(\sinh (\alpha)-\alpha \cosh (\alpha))+\mu_{0}\left(\sinh (\alpha)-\alpha \cosh (\alpha)+\alpha^{2} \sinh (\alpha)\right)}{\mu_{s}(\sinh (\alpha)-\alpha \cosh (\alpha))-\mu_{0}\left(\sinh (\alpha)-\alpha \cosh (\alpha)+\alpha^{2} \sinh (\alpha)\right)} \\
\alpha=R_{s} \sqrt{j \omega \mu_{\mathrm{s}} \sigma_{s}}
\end{gathered}
$$

The equations presented in this section have a two-fold purpose. First, it is apparent that the net effect is that the induced magnetic moments $\left(m_{p}\right.$ and $\left.m_{s}\right)$ act like superimposed point dipoles that generate a new magnetic field. This provides an abstraction to help interpret numerical simulations from a quantitative point of view. Secondly, the equations demonstrate that the induced magnetic dipoles are nonlinearly related to the material properties and radius of the object and shield in addition to the thickness of the shield. Although this nonlinearity inherently adds complexity, it is also a promising feature that permits inferring material properties via nondestructive methods. $H$ and $H_{s}$ correspond to the total magnetic field in the presence of the hidden object and to the scattered magnetic field in the absence of the hidden object. The z-directional magnetic fields are employed because the normal components of the fields are dominant for nondestructive evaluation $[8,19]$. For nondestructive evaluation, $H_{S}$ is calculated by Equation (7) where $S E_{H}$ is magnetic shielding effectiveness.

$$
\begin{gathered}
H_{s}=\frac{H_{0}}{S E_{H}}, \\
S E_{H}=\cosh \left(k d_{s}\right)+\frac{1}{3}\left(\frac{k R_{s}}{\mu_{r}}+\frac{2 \mu_{r}}{k R_{s}}\right) \sinh \left(k d_{s}\right), \\
S E_{H, B \& T}=1+\frac{1}{3} j w \mu_{0} \sigma_{s} d_{S} R_{s},\left(\text { if } \frac{k R_{s}}{\mu_{r}} \gg 1 \text { and } k d_{s} \ll 1\right),
\end{gathered}
$$


The $S E_{H}$ is expressed by Equation (8) where $k$ is a complex wave number $[7,20]$. The $S E_{H}$ in Equation (8) can be simplified to $S E_{H, B \& T}$ by Equation (9) when the radius of the shell is big and the thickness of the shell is narrow.

Figure 2 shows the induced magnetic dipole moment (in-phase component) as calculated by Equations (1)-(3). In Equation (1), $H_{0}$. is $1 \mathrm{~A} / \mathrm{m}$. The amplitude and polarity of an induced magnetic dipole $\left(m_{p}\right)$ are calculated as a function of relative permeability and radius of a sphere object. The incident magnetic field is assumed to be a uniform magnetic field, and the frequencies are $50 \mathrm{~Hz}, 200 \mathrm{~Hz}$, and $1 \mathrm{kHz}$ in the range of ELF/VLF signal. In the $50 \mathrm{~Hz}$ case, the induced dipole is positive in all radius cases at $\mu_{r}=1$. When the relative permeability increases, the polarity of the induced dipoles is changed from positive to negative, and the object can be considered as a ferromagnetic material. When the radius is incrementally increased from 0.1 to $0.4 \mathrm{~m}$, the amplitude of the induced dipole proportionally increases, and the slope of decreased $m_{p}$ is steeper. For example, the difference between $\mu_{r}=1$ and $\mu_{r}=1000$ at $R=0.4 \mathrm{~m}$ is 4.5 times higher than at $R=0.1 \mathrm{~m}$. When the incident field frequency increases, the amplitude of the induced dipole $\left(m_{p}\right)$ increases but the slope of the changes as a function of relative permeability slowly decreases. At $200 \mathrm{~Hz}$ and $1 \mathrm{kHz}$ with $\mu_{r}=10$ and $\mu_{r}=100$, the sphere object with $0.4 \mathrm{~m}$ radius has positive induced $m_{p}$ and is considered as a conductive material. In all cases, there is a critical permeability at which the dipole moment switches signs. This critical value also typically increases with increasing frequencies. Physically, at low values of permeability, the conductivity tends to dominate, and the induced fields are primarily due to Eddy currents. In contrast, for high values of permeability, the magnetization of the object dominates the response, and the Eddy currents are negligible. When the induced fields of an object are dominated by Eddy currents (low permeability), the induced field will oppose the incident field via application of Lenz's law. However, for a magnetization dominated object, the induced fields will in fact enhance the incident fields. As such, this is an important qualitative feature, since it can be readily utilized to infer properties about a hidden object without significant signal processing.
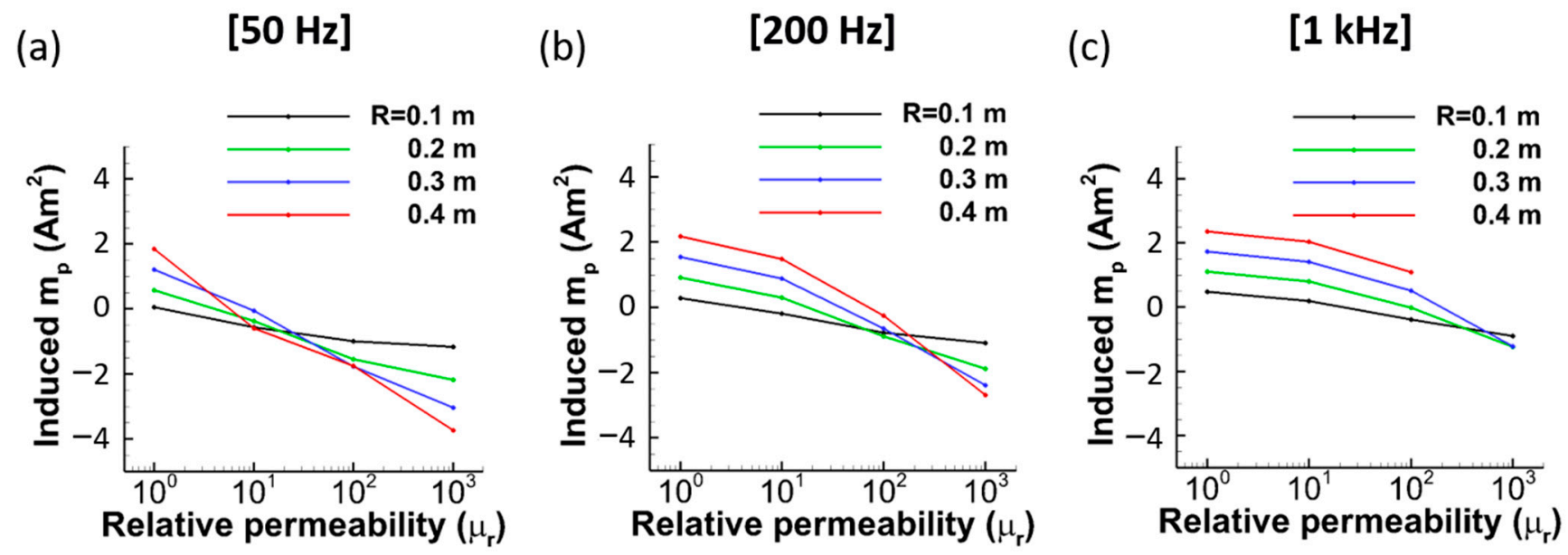

Figure 2. The amplitude $\left(m_{p}\right)$ of induced magnetic dipoles on sphere objects with various relative permeability at $50 \mathrm{~Hz}$, $200 \mathrm{~Hz}$, and $1 \mathrm{kHz}$. A radius of the sphere object is verified from 0.1 to $0.4 \mathrm{~m}$.

Figure 3 shows the $H$ fields and induced surface current for (a) conductive and (b) ferromagnetic cylinders in the presence of a $50 \mathrm{~Hz}$ magnetic dipole transmitter. The relative permeabilities of the conductive and ferromagnetic cylinders are 1 and 1000. The cylinders have a $0.2 \mathrm{~m}$ radius and $0.2 \mathrm{~m}$ height with $10^{6} \mathrm{~S} / \mathrm{m}$ conductivity. The center of the bottom of the cylinders is $x=y=z=0$. The incident magnetic field is assumed as a negative z-oriented uniform and perpendicular magnetic field on cylinder materials. Figure $3 a$ shows that a counterclockwise directional surface current on the conductive cylinder is induced. With the induced Eddy current, the illuminating magnetic field is blocked, 
and the field amplitude is reduced $3 \mathrm{~dB}$ compared to around the corner of the cylinder radius $\left(x^{2}+y^{2}=0.2^{2}, z=0\right)$. However, Figure 3b shows that a clockwise directional surface current on the ferromagnetic cylinder is induced. The illuminating magnetic field is gathered and the field amplitude is enhanced $3 \mathrm{~dB}$ compared to the boundary area at $x^{2}+y^{2}=0.2^{2}$ and $z=0$. This result shows the important phenomenon that high conductivity and high permeability have opposing effects. Specifically, a purely conductive object results in a magnetic depletion; i.e., the object essentially blocks the incident magnetic field. However, a purely permeable object focuses the field and creates an enhancement. The direction of the currents is dependent on a phase of the incident field in time and is opposite when a phase of the field is 180 degrees. For the non-magnetic object, the induced current has approximately the same phase as the incident field. For the magnetic object, the current is 180 degrees out of the phase with the incident field. For an object with mixed properties, this results in a complex dependence on the size, permeability, and conductivity of the object as alluded by Equation (2). However, the problem becomes considerably more complex with the introduction of an external shield.

(a)

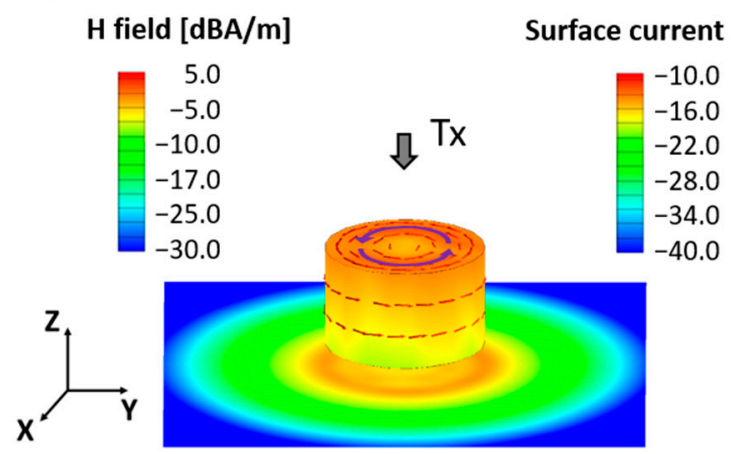

(b)

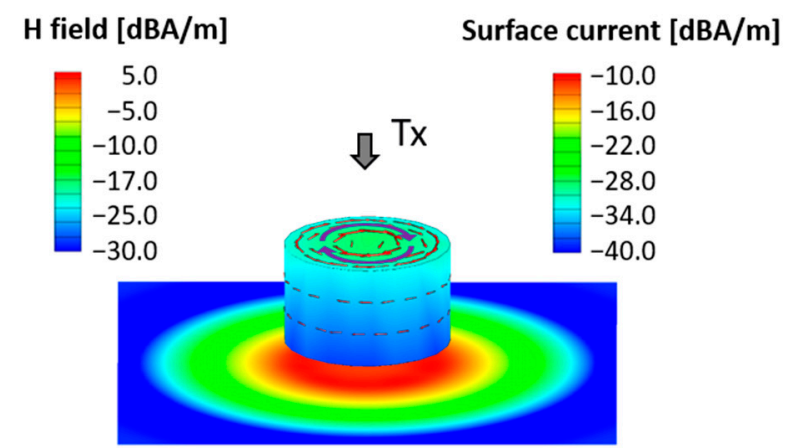

Figure 3. $H$ field and surface current with a different relative permeability of (a) 1 and (b) 1000 for cylinder materials at $50 \mathrm{~Hz}$ case. All cylinder materials have $10^{6} \mathrm{~S} / \mathrm{m}$ conductivity with a $0.2 \mathrm{~m}$ radius and height. The center of the cylinder at the bottom $(z=0)$ is $x=y=0$.

\section{Simulation Setups and Results \\ 3.1. Simulation Setups}

The most commonly used numerical techniques to calculate electromagnetic radiation are finite different time domain (FDTD), finite element method (FEM), and finite volume method (FVM) [21]. These techniques divide a system domain with a finite number of grids, elements, volumes in appropriate radiation boundary conditions such as Neuman, Dirichlet, and mixed boundary conditions [21]. The concepts are well suited for problems with inhomogeneous or cluttered media. However, the methods are highly computationally intensive and not ideally suited for thin shell shielded enclosures with a low surface area to volume ratio, because a large number of grid cells are required in the free space region inside the shield [7]. To reduce heavily computational time, a surface integral equation (SIE) can be derived. An advantage of the SIE method is that it is explicitly meshed and calculated on the surface of objects, not in the free-space region. For example, equivalent currents are only placed on the boundaries separating material regions. To avoid lowfrequency instability for applications of the SIE, an augmented Müller formulation of Maxwell's equations was suggested for stable very low frequency and very high contrast materials with the locally corrected Nyström (LCN) method [7,22]. Recently, the LCN method of the SIE was shown to match the experimental data of nondestructive shielding effectiveness with $3 \mathrm{~dB}$ [7].

In this paper, we employ a commercial electromagnetic software, FEKO [23]. We apply a method of moments (MoM) that is a full-wave solution of Maxwell's surface integral equations (SIE) in the frequency domain. 
Figure 4 shows an illustration of FEKO simulation setup with a hidden box inside a thin-shelled cubic shield. The system is illuminated by an infinitesimal point magnetic dipole that is oriented in the negative $\mathrm{z}$ direction with amplitude $1 \mathrm{Am}^{2}$. The side lengths of the outside shell are set at $1.2 \mathrm{~m}$, and the hidden box is located at $x=y=0.6 \mathrm{~m}$. The thickness of the outside shell is $2.7 \mathrm{~mm}$. The size and location of the outside shell are fixed in all cases. The outside shell was utilized and designed in recent previous experiments $[7,8]$. The transmitting magnetic dipole is located at $x=y=0.6 \mathrm{~m}$ and $z=1.2 \mathrm{~m}$. The frequency of the dipole is $50 \mathrm{~Hz}$, which is on the low end of the ELF/VLF band. Fifty and $60 \mathrm{~Hz}$ are commonly used by much of the world as power line operational frequencies. Utilizing $50 \mathrm{~Hz}$ allows us to indirectly investigate the potential of using background power line signals for ELF imaging. Furthermore, the $50 \mathrm{~Hz}$ frequency most clearly shows the hidden objects because of the inherently high degree of penetration [8]. The $200 \mathrm{~Hz}$ and $1 \mathrm{kHz}$ cases also have similar qualitative field profiles due to distortions from the hidden objects; however, the incident signal-to-noise ratio (SNR) is notably higher. All electromagnetic quantities are assumed to be time harmonic $\left(e^{j w t}\right)$ and all data are calculated when the source field has zero phase $(t=0)$. The receivers are assumed to be located on the light blue plane below the box and parallel planes below it. The locations of the transmitter, center of the inside box, and outside shell are fixed in all cases. The simulation utilizes 8770 surface elements on the box and the observation has $1 \mathrm{~cm}$ grid resolution.

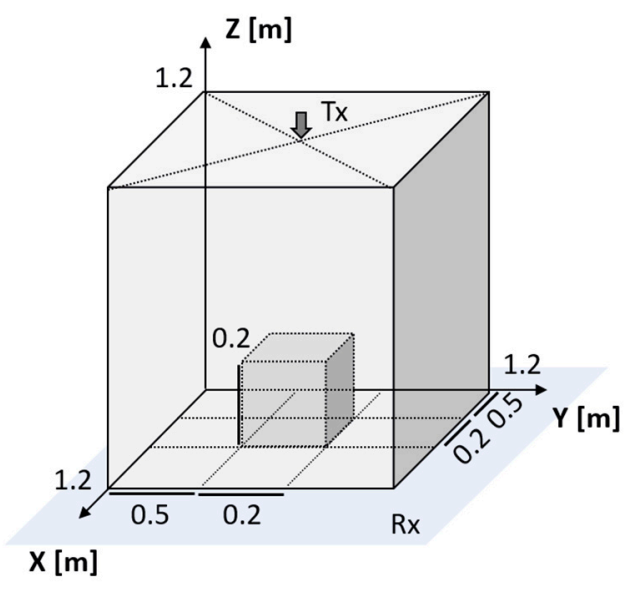

Figure 4. Illustration of simulation setup of an inside box and outside box shield in the near-field of an infinitesimal point magnetic dipole. $T_{x}$ and $R_{x}$ represent a transmitter of the dipole and receiver. The receivers are located on the light blue plane and negative $\mathrm{z}$-direction. The thickness of the outside shell is $2.7 \mathrm{~mm}$.

\subsection{Results}

To examine the relative effects of the shield, the gain $G$ is expressed by $G=20$ * $\log \left(\frac{\left|H_{s} \hat{\imath}\right|}{\left|H_{0} \imath\right|}\right) \mathrm{dB}$, which is the ratio of the scattered field in the absence of the hidden object to the total field in the presence of the hidden object. Figure 5 shows the gain $\mathrm{G}$ for different sizes of the hidden box as a function of spatial coordinates. For this set of simulations, the hidden object is assumed to be a perfect electrical conductor (PEC) in the shape of a cube. The conductivity of the outside shell is $10^{6} \mathrm{~S} / \mathrm{m}$, and the distortion magnetic fields are measured at the negative $z$-direction. Figure 5 a shows the gain fall-off with receiver distance for different widths and lengths of the hidden PEC box with the same height. Specifically, the gain is measured at $x=y=0.6 \mathrm{~m}$ (central axis of the system). The gain decreases with the receiver stand-off distance with an $\frac{1}{r^{3}}$ profile as expected from that of a magnetic dipole; however, notable differences are observed for the different sized objects. For instance, at $z=-0.005 \mathrm{~m}$, when the length $(y)$ increases from 0.2 to $0.4 \mathrm{~m}$, the $\mathrm{G}$ value increases by $2 \mathrm{~dB}(29.9$ to $31.9 \mathrm{~dB})$. When the area of the inside box is the same $(x 0.2 \mathrm{~m}$, y $0.4 \mathrm{~m}$ vs. $x 0.4 \mathrm{~m}, y 0.2 \mathrm{~m}$ ), the gain $G$ values are almost the same. When the width and length increase from 0.2 to $0.4 \mathrm{~m}$, the $G$ value increases 1.52 times (29.9 to $45.4 \mathrm{~dB})$. At 
$z=-1 \mathrm{~m}$, the $\mathrm{G}$ value for the $0.04 \mathrm{~m}^{2}$ area is 4.6 times higher $(0.0327$ to $0.151 \mathrm{~dB})$ than in the case of the $0.16 \mathrm{~m}^{2}$. As the stand-off distance of the receiver is increased (negative $\mathrm{z}$-direction), the difference in gain between the different conditions is accentuated. This is consistent with the theoretical expectations from Section 2 where the induced magnetic dipole moment is proportional to the volume of the hidden object and the corresponding fields fall off as the cubic distance from the object's location.

(a)

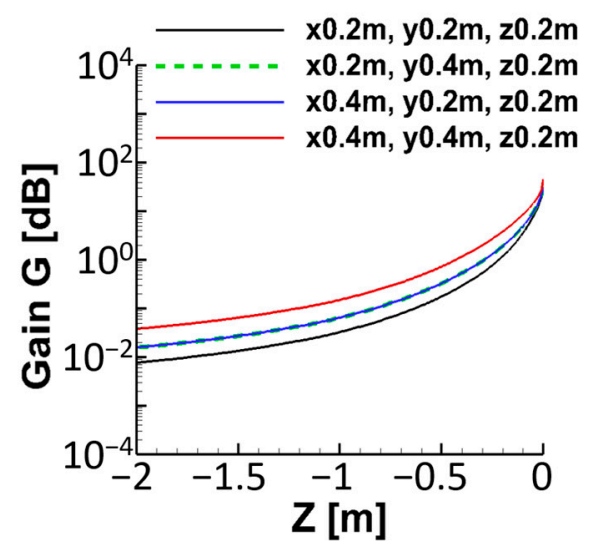

(b)



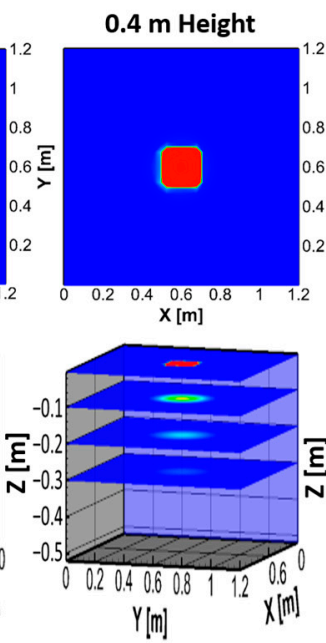

$Y[m]$

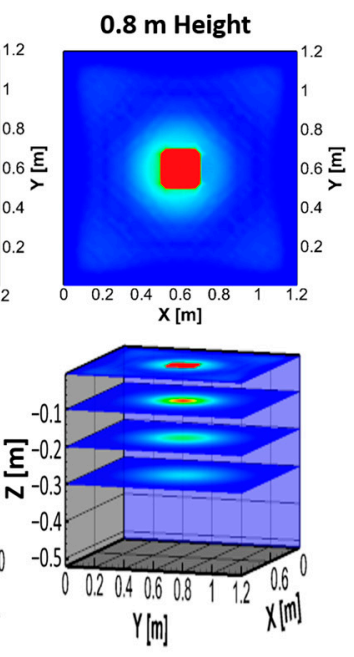

Figure 5. The G values for (a) different sizes of PEC inside box as a function of width (x), length (y), and height (z). (b) 0.2, 0.4 , and 0.8 height with 0.2 width and 0.2 length on $x y$ plane at $z=-0.005 \mathrm{~m}$ and $x y z$ geometry. The relative permeability of the inside box is fixed at 1 .

Figure $5 \mathrm{~b}$ shows the spatial gain profile for three different heights of the hidden box (from 0.2 to $0.8 \mathrm{~m}$ ). Note that the spatial profile is shown in the region everywhere below the external shield (negative $\mathrm{z}$ ). The cross-sectional areas of the three boxes at $z=-0.005 \mathrm{~m}$ have the same highest $G$ values in all conditions, as shown in Figure 5b. However, the gain increases and spreads out at the greater height of the object. This is an important feature since it demonstrates that the field distortion is sensitive to the height of a hidden object and not simply its cross-section, which allows investigating features of hidden objects that are "deep" inside the enclosure. This is also consistent with the theoretical expectations that induced magnetic dipole moment on the object is proportional to the volume of the object.

The results shown in Figure 5 suggest that many of the basic analytical features described in Section 2 still hold well even when the geometry of the hidden objects and shield deviate from that of a sphere. This provides support for developing simplified and computationally feasible inversion algorithms for non-destructive evaluation using ELF/VLF magnetic fields.

The results shown in Figure 5 demonstrate that the field distortions (or gain) are sensitive to the size of the hidden object inside an enclosure. However, different passive electrical properties of the shield can also induce different magnetic distortion fields. Figure 6 shows the $G$ value for different conductivities of inside and outside boxes for $50 \mathrm{~Hz}$ magnetic fields. The relative permeability of all cases is fixed at 1 . Figure $6 \mathrm{a}-\mathrm{c}$ show the gain profile for an enclosure with the conductivity of $10^{6}, 10^{7}$, and $10^{8} \mathrm{~S} / \mathrm{m}$, respectively; the hidden object is once again assumed to be a PEC cube which, has a length of $0.2 \mathrm{~m}$ with the center of the PEC. The thickness and size of the shell are the same as shown in Figure 4. The $\mathrm{G}$ values are measured on $x y$ plane at $z=-0.005 \mathrm{~m}$. The difference between the maximum and minimum of the $G$ values is $15 \mathrm{~dB}$. When the conductivity increases from $10^{6}$ to $10^{7} \mathrm{~S} / \mathrm{m}$, the $\mathrm{G}$ values are almost the same. The location and area of the PEC inside box are easily discernable. However, at $10^{8} \mathrm{~S} / \mathrm{m}$, the image of the inside box is slightly distorted from that of the lower conductivity shield cases. This is an interesting feature as 
(a)

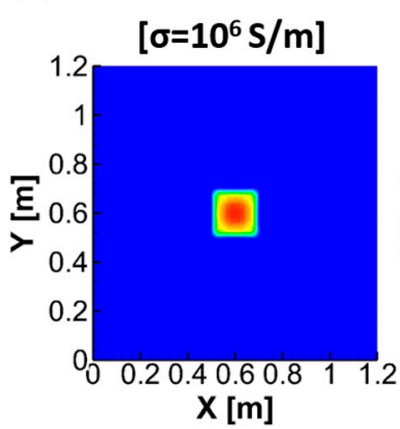

it indicates a threshold conductivity requirement before the fields are appreciably distorted on the observation plane.

(b)

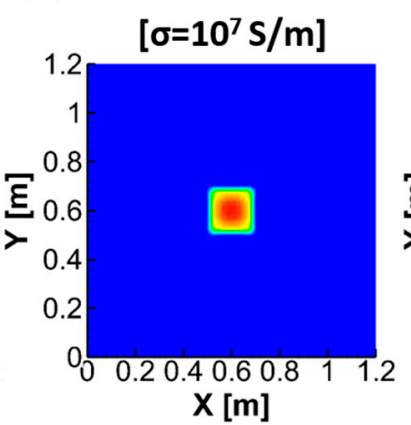

(c)

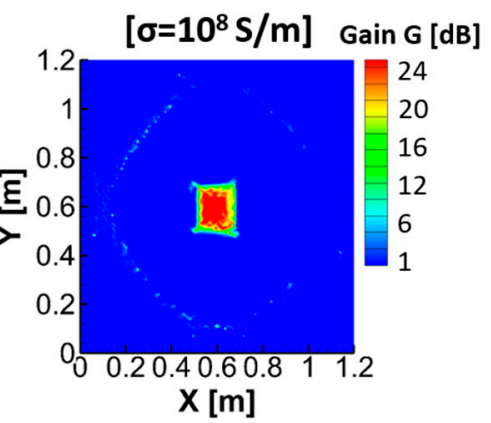

(d)



Figure 6. The $\mathrm{G}$ values for different conductivities of inside and outside boxes at $50 \mathrm{~Hz}$ magnetic fields. (a-c) Different conductivities of the outer shell for PEC inside box. (d) Different conductivities of the inside box through an outer shell that has $10^{6} \mathrm{~S} / \mathrm{m}$ conductivity. The relative permeability of all cases is 1 .

Figure $6 \mathrm{~d}$ shows the gain when the conductivities of the hidden box are changed but the outer shell has a fixed $10^{6} \mathrm{~S} / \mathrm{m}$ conductivity. The $\mathrm{G}$ value is measured at the center of the $x y$ plane along the negative $z$-direction. In the PEC case, the $G$ value is the highest ( $29.6 \mathrm{~dB}$ ) and sharply decreases along the negative $\mathrm{z}$-direction. Far from $z=-0.02 \mathrm{~m}$, the cases of PEC and $10^{8} \mathrm{~S} / \mathrm{m}$ conductivity have the same $\mathrm{G}$ value. When the conductivity of the inside box decreases from $10^{8}$ to $10^{7} \mathrm{~S} / \mathrm{m}$, the $\mathrm{G}$ value is reduced from 19.2 to $0.9 \mathrm{~dB}$. For a conductivity of $10^{6} \mathrm{~S} / \mathrm{m}$, the $\mathrm{G}$ value is 0 . This suggests that the object is not easily detectable when the conductivity of both inside and outside boxes is the same. This suggests that ELF/VLF magnetic fields have difficulty discerning hidden objects when the conductivity contrast is not appreciably high and is an important limitation of the method.

Figure 7 shows that the $H$ fields are measured on the $x y$ plane at $z=-0.005 \mathrm{~m}$ for conductive $\left(\mu_{r}=1\right)$ and ferromagnetic $\left(\mu_{r}=1000\right)$ materials through an outer shell at $50 \mathrm{~Hz}$ case. The inside objects in cube geometry have a length of $0.6 \mathrm{~m}$ with $\sigma_{\text {in }}=10^{6-8} \mathrm{~S} / \mathrm{m}$ conductivity. The outer shells have $2.7 \mathrm{~mm}$ thickness with $\sigma_{\text {out }}=10^{6-8} \mathrm{~S} / \mathrm{m}$ conductivity. The size of the outer shells and the location of magnetic dipoles are the same as shown in Figure 4. Compared to only inside materials in Figures 2 and 3, there is a conductive outer shell. When an outer shell has lower conductivity than an inside object, the size and location of the inside object can be imaged as shown in Figure $7 \mathrm{e}, \mathrm{f}, \mathrm{i}, \mathrm{j}$. From the boundary of the inside box, the magnetic fields decrease when the measurement point goes to the center $(x=y=0.6 \mathrm{~m})$.

The reason is that a negative-induced dipole induces an enhanced magnetic field with gathered magnetic flux as shown in Figures 2 and 3 . However, at $\sigma_{\text {in }}=10^{7} \mathrm{~S} / \mathrm{m}$, the magnetic field in the case of the ferromagnetic material increases again as the area of measurement is less than a half in the radius of the object $\left((x-0.6)^{2}+(y-0.6)^{2}<0.3^{2}\right)$, as shown in Figure 7f. At $\sigma_{\text {in }}=10^{8} \mathrm{~S} / \mathrm{m}$, the $H$ field continually decreases toward the center, but the amplitude is enhanced compared to the conductivity material case, as shown in Figure 7i,j. When the conductivities of the inside box and outer shell are the same, both boxes can be considered as an unseparated one box, as shown in Figure $7 \mathrm{a}, \mathrm{b}$. At $\sigma_{\text {out }}=10^{8}$, the location and area of the hidden PEC box are easily discernable in Figure $6 c$, but the inside materials with $\sigma_{i n}=10^{6-8} \mathrm{~S} / \mathrm{m}$ cannot be imaged because of less distortion field.

As shown in Figure 2, the area of the enhanced or reduced magnetic field is dependent on applied frequency and material properties. The location of receivers also needs to be considered to investigate the area of the enhanced field in the future. 

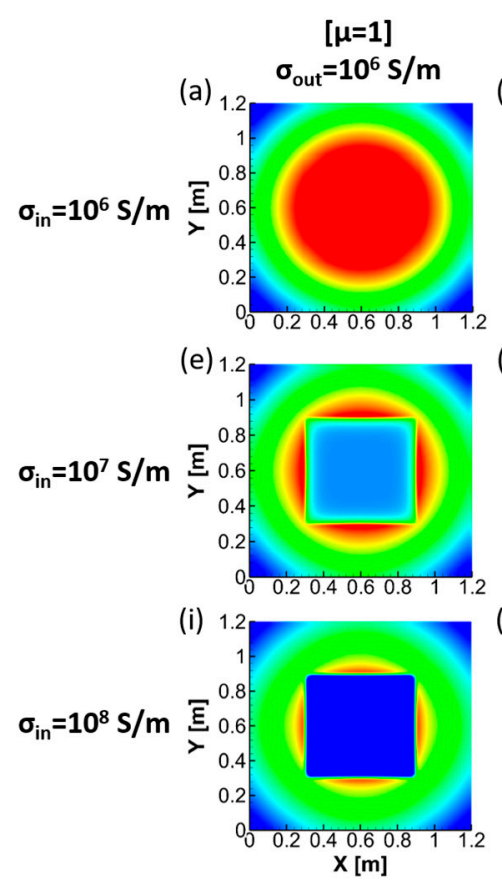
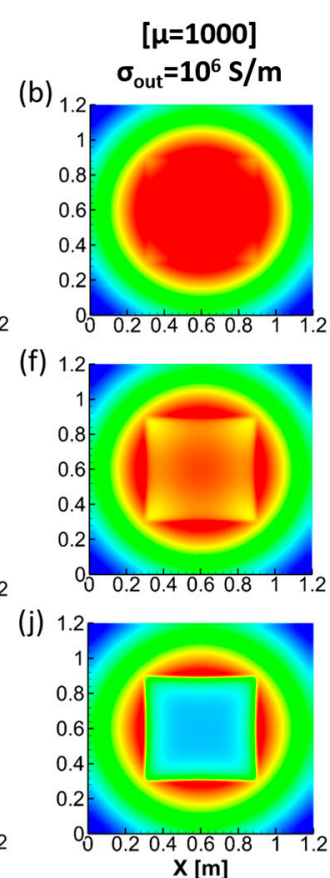
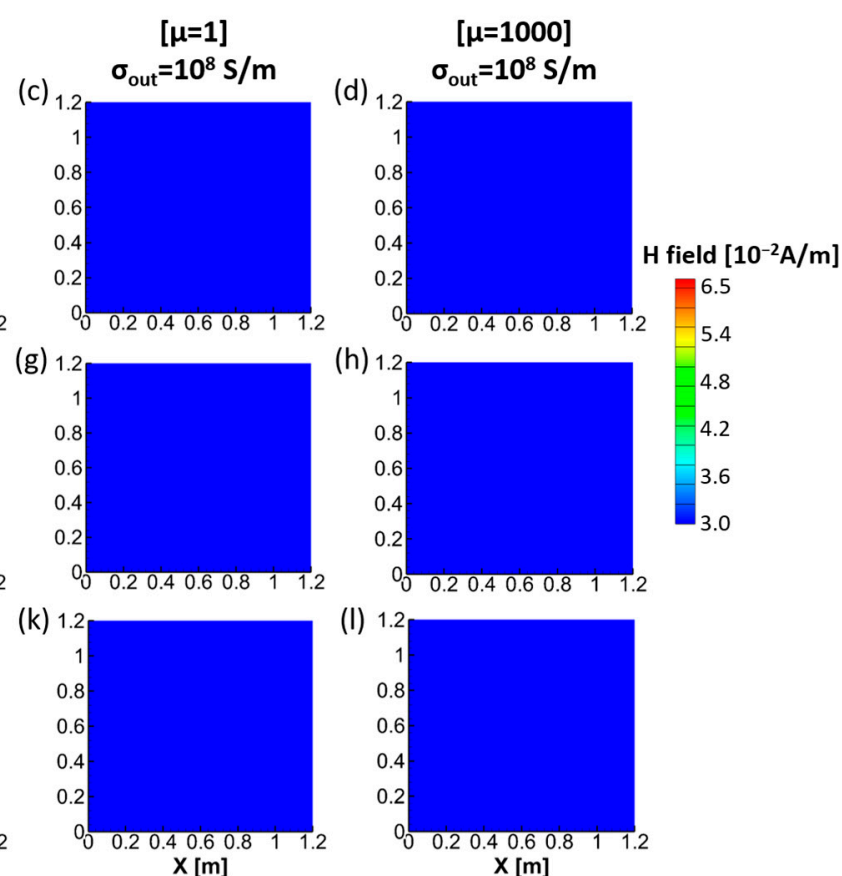

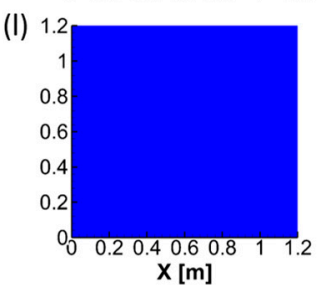

Figure 7. $H$ fields on $x y$ plane at $z=-0.005 \mathrm{~m}$ for $(\mathbf{a}, \mathbf{c}, \mathbf{e}, \mathbf{g}, \mathbf{i}, \mathbf{k})$ conductive $\left(\mu_{r}=1\right)$ material and $(\mathbf{b}, \mathbf{d}, \mathbf{f}, \mathbf{h}, \mathbf{j}, \mathbf{l})$ ferromagnetic $\left(\mu_{r}=1000\right)$ material through an outer shell at $50 \mathrm{~Hz}$ signal. The conductivities of the outer shell and inside box are in the range of $10^{6}$ to $10^{8} \mathrm{~S} / \mathrm{m}$. The inside object in cube geometry has lengths 0.6 with the center and the outer shell has $2.7 \mathrm{~mm}$ thickness.

\section{Conclusions and Discussion}

Nondestructive evaluation of various conductive objects through metal enclosures is investigated by using ELF/VLF magnetic fields. In the presence of an incident field, the hidden objects create field distortions that can be measured outside the enclosure. The field distortion is primarily observed for the normal component of the field on the shield surface and is quantified using the gain G. The gain is shown to be sensitive to the size, conductivity, and permeability of objects, which could provide support for imaging applications. The G value is shown to decrease with increasing distance of the measurement plane from the base of the hidden object with a spatial profile that is consistent with simplified analytical theory. As shown in Figure 5, the projection of the fields on different measurement planes is quite sensitive to the distance from the base of the box. It is apparent that the distance of the measurement plane is an important parameter in determining the spatial resolution on the $x y$ plane. In general, the closer the measurement is to the object, the higher the lateral resolution. As the distance of the measurement plane is increased, the fields will more closely approximate that of a single spherical object, and high spatial resolution will become difficult to attain.

When the conductivities of the outer shell are $10^{6}$ to $10^{8} \mathrm{~S} / \mathrm{m}$, the imaged size of the PEC box is almost the same as the actual PEC box size. Several actual materials lie in the range of conductivities utilized in this study. Some representative materials and corresponding conductivity values are nickel $\left(1.28 \times 10^{6} \mathrm{~S} / \mathrm{m}\right)$, aluminum $\left(3.5 \times 10^{7} \mathrm{~S} / \mathrm{m}\right)$, copper $\left(5.8 \times 10^{7} \mathrm{~S} / \mathrm{m}\right)$, and silver $\left(6.3 \times 10^{7} \mathrm{~S} / \mathrm{m}\right)$ [17]. The $\mathrm{G}$ value decreases when the conductivities of the inside box decrease $10^{8}$ to $10^{6} \mathrm{~S} / \mathrm{m}$. The distorted field vanishes at far from $z=-0.3 \mathrm{~m}$. It is shown that objects with conductive materials and low permeability tend to induce a "magnetic field shadow" below the object. In contrast, high permeability materials tend to show an enhancement in the magnetic field at a measurement location below the object.

These opposed results also show nondestructive evaluation through a conductive outer shell. The polarity of the surface current is dependent on the radius of sphere material, frequency, and relative permeability. The difference of both conductivities in the inside 
and outer shell are also considered. A hidden object can be detected as the conductivity of the inside object is higher than that of the outer enclosure. The results can be utilized for the practical and fundamental limits to nondestructive imaging of conductive objects through metal enclosure using ELF/VLF magnetic fields. Several practical considerations should be kept in mind when selecting the appropriate frequency (or frequency range) for specific applications. The first relevant variable involves information on material properties within the application at hand. For instance, if imaging inside enclosures akin to a shipping container is desired, the frequency must be chosen to be low enough to penetrate the material but not so low that the response of hidden objects is not observable (this will likely be in the sub $100 \mathrm{~Hz}$ range). A second important variable is the noise characteristics of the application environment. In the ELF/VLF band, the dominant sources of noise are atmospheric noise (typically from lightning radiation), nearby electronics, and power line harmonics. Unless any of the "noise" signals are directly utilized as signals of opportunity, frequencies that are not heavily interrupted by noise are likely the best choice to maximize the SNR of scattered signals. A third important parameter is the type of sensor being utilized. The most common type of ELF/VLF sensors is loop antennas [24] that typically lose sensitivity as frequency is decreased. Thus, frequencies that are too low may not be easily detectable using standard sensors or may require either specialty or expensive equipment.

In general, the frequency response of a hidden object and shield combination can be relatively complex. As such, the use of multiple frequencies is likely the most prudent methodology in practice. As a qualitative description, higher frequencies tend to achieve less penetration into a shielded container, but with the advantage of responding more to conductivity than permeability. On the other hand, the lower frequencies demonstrate a high degree of penetration and are considerably more sensitive to magnetic properties (permeability) relative to higher frequencies. For the purposes of this work, the $50 \mathrm{~Hz}$ frequency case was chosen based on a relatively good (empirically determined) trade-off between penetration and sensitivity to material properties and also due to the ubiquity of $50 \mathrm{~Hz}$ radiation from global power line networks.

The time-domain signature of the magnetic field distortions plays an important role in imaging. However, the simulations in this case are run applying monochromatic excitation to ascertain the response to low frequencies in a more straightforward manner. Thus, any time-variation is encoded in the amplitude and phase profile of the measured signals. It is worth noting that this method can be extended to the time-domain in future work by running several frequencies and synthesizing the time-domain signal. The results of the work provide a basis for future inversion methodologies for imaging objects through shielded enclosures using ELF/VLF signals.

Author Contributions: Conceptualization, H.K.; methodology, V.H.; software, H.K.; validation, V.H.; formal analysis, H.K.; investigation, H.K., V.H.; writing—original draft preparation, H.K.; writingreview and editing, V.H.; project administration, V.H.; funding acquisition, V.H. All authors have read and agreed to the published version of the manuscript.

Funding: This research was funded by the Defense Advanced Research Projects Agency (DARPA), Imaging Through Almost Anything Anywhere (ITA3) grant number HR00111820016.

Institutional Review Board Statement: Not applicable.

Informed Consent Statement: Informed consent was obtained from all subjects involved in the study.

Data Availability Statement: All data are available on Github (https:/ / github.com/hoyoungrkim/ Numerical-modeling-of-nondestructive-testing-of-various-con-ductive-objects-inside-metal-enclosures, accessed on 19 April 2021).

Conflicts of Interest: The authors declare no conflict of interest. 


\section{References}

1. Cummer, S.A.; Inan, U.S.; Bell, T.F. Ionospheric D region remote sensing using VLF radio atmospherics. Radio Sci. 1998, 33, 1781-1792. [CrossRef]

2. Golkowski, M.; Sarker, S.R.; Renick, C.; Moore, R.C.; Cohen, M.B.; Kulak, A.; Miynarczyk, J.; Kubisz, J. Ionospheric D region remote sensing using ELF Sferic group velocity. Geophys. Res. Lett. 2018, 12, 739-748. [CrossRef]

3. Kim, H.; Harid, V. Hybrid-PIC Simulation of Nonlinear Wave-Particle Interactions in the Earth's Radiation Belts. In Proceedings of the International Applied Computational Electromagnetics Society Symposium (ACES), Miami, FL, USA, 14-19 April 2019; pp. 1-2.

4. Wait, J.R. Theory of magnetotelluric fields. J. Res. Nat. Bureau Stand. D 1962, 66, 509-541. [CrossRef]

5. Golkowski, M.; Harid, V.; Hosseini, P. Review of controlled excitation of non-linear wave-particle interactions in the magnetosphere. Front. Astron. Space Sci. 2019, 6, 1-20. [CrossRef]

6. Rowe, H.E. Extremely low frequency (ELF) communication to submarines. IEEE Trans. Commun. 1974, 22, 371-385. [CrossRef]

7. Harid, V.; Golkowksi, M.; Gedney, S.D.; Cohen, M.B.; Patch, S.K.; Rorrer RA, L.; Renick, C.M.; Bittle, J.; Opalinski, N.M.; Kim, H.Y. Magnetic Field Penetration Into a Metal Enclosure Using an ELF/VLF Loop Antenna. IEEE Trans. Electromagn. Compat. 2019, 62, 1225-1236. [CrossRef]

8. Kim, H.Y.; Harid, V.; Mroz, A.; Wewerka, J.; Todorovski, D.J.; Golkowski, M.; Rorrer RA, L.; Cohen, M.B.; Opalinski, N.M. Imaging Conductive Objects Through Metal Enclosures Using ELF/VLF Magnetic Fields. IEEE Access 2020, 8, 79745-79753. [CrossRef]

9. Korjenevsky, A.; Cherepenin, V.; Sapetsky, S. Magnetic induction tomography: Experimental realization. Physiol. Meas. 2000, 21, 89-94. [CrossRef] [PubMed]

10. Soleimani, M. Super-sensing through industrial process tomography. Philos. Trans. R. Soc. A 2016, 374, 20150445. [CrossRef] [PubMed]

11. Liu, R.; Li, Y.; Fu, F.; You, F.; Shi, X.; Dong, X. Time-difference imaging of magnetic induction tomography in a three-layer brain physical phantom. Meas. Sci. Technol. 2014, 25, 065402. [CrossRef]

12. Ma, L.; Soleimani, M. Magnetic induction tomography methods and applications: A review. Meas. Sci. Technol. 2017, 28, 1-12. [CrossRef]

13. Mansor, M.S.B.; Zakaria, Z.; Balkhis, I.; Rahim, R.A.; Sahib, M.F.A.; Yunos, Y.M.; Sahlan, S.; Bunyamin, S.; Abas, K.H.; Ishak, M.H.I.; et al. Magnetic induction tomography: A brief review. J. Teknol. 2015, 73, 91-95.

14. Todorovski, D.J. Low-Frequency Imaging Algorithm for Inverting Arbitrary Electromagnetic Systems. Master's Thesis, University of Colorado Denver, Denver, CO, USA, 2019.

15. Merwa, R.; Hollaus, K.; Brunner, P.; Scharfetter, H. Solution of the inverse problem of magnetic induction tomography (MIT). Physiol. Meas. 2005, 26, S241-S250. [CrossRef] [PubMed]

16. Wei, H.Y.; Soleimani, M. Three-dimensional magnetic induction tomography imaging using a matrix free krylov subspace inversion algorithm. Prog. Electromagn. Res. 2012, 122, 29-45. [CrossRef]

17. Won, I.J.; Keiswetter; Bell, T.H. Electromagnetic Induction Spectroscopy for Clearing Landmines. IEEE Trans. Geosci. Remote Sens. 2001, 39, 703-709. [CrossRef]

18. Hill, D.A.; Wait, J.R. The electromagnetic response of a buried sphere for buried-dipole excitation. Radio Sci. 1973, 8, 813-818. [CrossRef]

19. Einaudi, F. Shielding of electromagnetic waves by a spherical shell. J. Appl. Phys. 1971, 42, 545-549. [CrossRef]

20. Hasselgren, L.; Luomi, J. Geometrical aspects of magnetic shielding at extremely low frequencies. IEEE Trans. Electromagn. Compat. 1995, 37, 3. [CrossRef]

21. Chung, T.J. Computational Fluid Dynamics; Combridge University Press: Combridge, UK, 2002; Chapter 1.

22. Hendijani, N.; Gedney, S.D.; Young, J.C.; Adams, R.J. A Nystrom discretization of a broad-band augmented-muller surface integral equation. ACES J. 2018, 33, 1060-1067.

23. EM Software and Systems, FEKO Suite 7.0. Available online: http://www.feko.info (accessed on 19 April 2021).

24. Cohen, M.B.; Inan, U.S.; Paschal, E.W. Sensitive broadband ELF/VLF radio reception with the AWESOME instrument. IEEE Trans. Geosci. Remote Sens. 2010, 48, 3-17. [CrossRef] 